\title{
DEVELOPMENT OF A MULTIPHASE FLOW MIXER DEVICE FOR PETROLEUM INDUSTRY
}

\author{
Lima, M. P. ${ }^{1}$; Martins, M. F. ${ }^{1}$ \\ ${ }^{1}$ Computational Transport Phenomena Laboratory - LFTC, Graduate Program in Mechanical Engineering, Federal \\ University of Espírito Santo - UFES, Av. Fernando Ferrari - 514, Goiabeiras, Vitória, ES, Brazil
}

\begin{abstract}
The use of secondary recovery methods for oil wells has become a common practice in the petroleum industry in order to increase production and ensure oil flow to the surface. However, the technological challenges faced, especially offshore, hamper oil rising and open up a range of opportunities for the development of new technologies and enhancement of existing ones. One of the main methods used in artificial lift is the Electrical Submersible Pump (ESP), which has presented problems in gassy well due to electric current fluctuations and gas lock occurrence in the system. The present work proposes the development of a device capable of reducing the negative impact of the large amount of gas mixed with oil in ESP systems by homogenizing the phases upstream the pump. Additionally, the equipment also seems promising in multiphase flow measurement thanks to its ability of reducing the influence of flow regimes along the measurement. CFD multiphase flow simulation was accomplished in commercial software to evaluate its performance in mixtures with high concentration of gas. The simulations results suggest a significant reduction of the gas fraction downstream the equipment to less than $20 \%$ for more than $70 \%$ of gas at the entry.
\end{abstract}

Keywords: CFD, Multiphase, ESP.

\section{Introduction}

The growth of world energy demand associated with the importance of oil in the global energy sources makes it necessary to ensure the supply of this raw material in the entire planet. Since new reserves discoveries have not kept pace with the increasing demand, the intensification of oil production in existing reserves becomes essential. The so called secondary recovery techniques, such as Gas Lift systems and Electrical Submersible Pumps (ESP), can be then applied to optimize production. Although ESP systems are widely used in the industry, it has been found that, for gassy wells, the pump operates badly due to alternating free gas and heavy fluid flow, as remarked by [1]. Over the years, many companies have developed technologies intending to enhance ESP efficiency for high gas concentration flow. Schlumberger [2] claims that, by homogenizing the mixture upstream the ESP, its Poseidon Multiphase Gas-Handling device can handle up to $75 \%$ of free gas against $20 \%$ tolerated by conventional pumps without this technology.
In another context, gas-liquid multiphase flow measurements are also challenging since this sort of mixture admits different flow regimes inside pipes depending on its dimensions and volume fraction of both phases. This feature is presented by [3] to be a concern in multiphase measurements results. To minimize the fluctuating results obtained due to the heterogeneous passage of different phases through the meter device, the homogenization of the mixture can then be performed before the measurement.

In the present work, CFD simulation of a new multiphase flow mixer technology is carried out by Volume of Fluid (VOF) model in Fluent software. The study shows the device capacity of homogenizing a gas-liquid mixture with high gas volume fractions and a validation of the model.

\section{CFD modeling}

\subsection{VOF model}

According to [4], in Volume of Fluid (VOF) model, two or more not interpenetrating continuous phases are 
simulated by using one continuity equation (Eq. 1) for each phase and a single momentum equation (Eq. 2) with a shared velocity field among the phases:

$$
\begin{gathered}
\frac{\partial \alpha_{q} \rho_{q}}{\partial t}+\nabla \cdot\left(\alpha_{q} \rho_{q} \vec{v}_{q}\right)=M_{q} \\
\frac{D(\rho \vec{v})}{D t}=-\nabla p+\nabla \cdot\left[\mu\left(\nabla \vec{v}+\nabla \vec{v}^{T}\right)\right]+\rho \vec{g}+\vec{F}
\end{gathered}
$$

where, respectively, $\alpha_{q}, \rho_{q}$ and $\vec{v}_{q}$ are volume fraction, density and velocity of phase $q ; \vec{v}, p, \vec{g}$ are velocity, pressure and gravity shared among the phases; $\mu$ and $\rho$ are viscosity and density of the mixture, defined as weighted average based on $\alpha_{q} ; \quad M_{q}$ and $\vec{F}$ are source terms for mass (from others phases to phase $q$ ) and force. In the present study, no mass transfer is taken in account, but $\vec{F}$ is computed as surface force (Eq. 3) at the interface between phases:

$$
F=\sum_{i j, i<j} \sigma_{i j} \frac{\alpha_{i} \rho_{i} k_{j} \nabla \alpha_{j}+\alpha_{j} \rho_{j} k_{i} \nabla \alpha_{i}}{\frac{1}{2}\left(\rho_{i}+\rho_{j}\right)}
$$

where index $\boldsymbol{i}$ and $\boldsymbol{j}$ means arbitrary phases; $\alpha, \rho$, $k$ and $\sigma$ are, respectively, volume fraction, density, radius of curvature at the interface and surface tension, which is set as constant in the present study.

\subsection{VOF validation and device performance simulations} Since no prototype for the mixer device have been built in this first step of the development, no experimental validation was accomplished with its geometry, however the VOF model simulation was validated based on [5]. The geometry simulated was similar to the one used by [5], but a 5.395.183 cells unstructured mesh was performed. The same physical parameters and boundary conditions were also established. PISO pressure-velocity coupling algorithm with second-order upwind spatial discretization and explicit time discretization were set in the validation and device performance simulations, with convergence criteria equal to $10^{-7}$ for all parameters.

\section{Results and discussion}

In [5], vertical plug gas-liquid flow regime was established experimentally (Fig.1c) and numerically (Fig.1b). Both have been compared with the numerical result obtained in the present work (Fig.1a), and a good qualitative matching was achieved since similar bubble shape was obtained for all.

The Figure 2 shows a comparison between the gas volume fraction obtained in VOF simulation for a free bubble rising into a tube and for the same bubble after leaving the mixer device (both for given cross sections along the tube).

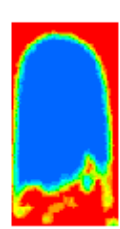

(a)

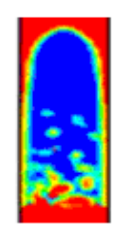

(b)

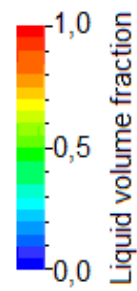

(c)
Figure 1: Results comparison between simulation performed in this work (a) and numerical (b) and experimental (c) results accomplished by [5].

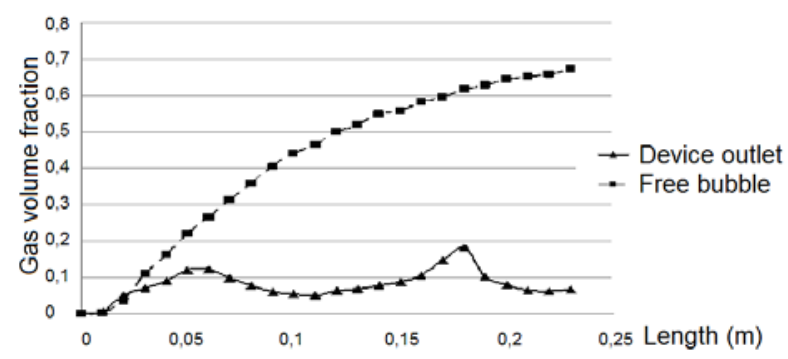

Figure 2: Gas volume fraction for each cross section of the tube without the mixer device (free bubble) and with it.

\section{Conclusions}

Since the plug regime is established in validation simulation and the bubble shape has a similar geometry to those obtained by [5], the VOF validation can be qualitatively confirmed.

According to the Figure 2, the mixer device is capable of prolonging gas distribution along the tube and reducing gas volume fraction to less than $20 \%$ when a free bubble rises into the pipe reaching almost $70 \%$ in volume fraction of gas.

\section{References}

[1] POWERS, W.J.; DUNBAR, C.; CHILINGARIAN, G. V. Electric Submergible Pumps. In: Surface Operations in Petroleum Production. New York: Elsevier Science Publishers., 1987. cap. 16, p. 737-799.

[2 ]http://www.slb.com/services/production/artificial lift/ submersible/esp_components/esp_gas_handling/posei don_gas_handling.aspx

[3] PALADINO, E. E. Estudo do escoamento multifásico em medidores de vazão do tipo pressão diferencial. 2005. 263 f. Tese (Doutorado em Engenharia Mecânica), Universidade Federal de Santa Catarina, Florianópolis, 2005.

[4] FLUENT, ANSYS FLUENT Theory Guide, ANSYS Inc., 2009.

[5] PARVAREH, A.; RAHIMI, M.; ALIZADEHDAKHEL, A.; ALSAIRAFI, A., Heat and Mass Transfer, v. 37, p. $304-311,2010$. 